

\title{
Desenvolvimento de manequins para o ensino de moldagens para confecções de prótese bucomaxilofacial intraoral: relato de experiência
}

\author{
Development of mannequins to teach shaping for the confection of intraoral \\ maxillofacial prosthesis: a report of experience
}

\author{
Ana Luiza Michel Costa \\ Centro Universitário Newton Paiva \\ Graduanda em Odontologia \\ analuiza.michel12@gmail.com \\ Kathleen Salzamann Castro \\ Centro Universitário Newton Paiva \\ Graduanda em Odontologia \\ k.salzmann@outlook.com.br \\ Fernando Barbosa Marques Porfírio \\ Centro Universitário Newton Paiva \\ Professor do curso de Odontologia \\ fernando_odontobh@hotmail.com \\ Frederico Santos Lages \\ Universidade Federal de Minas Gerais \\ Professor do curso de Odontologia \\ fredlages@hotmail.com
}

\section{RESUMO}

\begin{abstract}
A prótese bucomaxilofacial é a especialidade responsável pela reparação de imperfeições faciais intra e extra orais, congênitas ou adquiridas. Os defeitos faciais comprometem algumas funções biológicas, estéticas, e podem, também, causar danos psicológicos. É de suma importância a atuação de uma equipe multidisciplinar para analisar a etiologia do problema para o qual o paciente procura tratamento. Há um aumento expressivo na qualidade de vida dos pacientes usuários das próteses, indicando a importância da especialidade. O objetivo deste trabalho é, através de um relato de experiência, descrever o desenvolvimento de um manequim único e exclusivo para auxiliar o treinamento de moldagens de pacientes com imperfeições intraorais e, consequentemente, difundir o ensino da especialidade entre as faculdades de odontologia. Os manequins criados são utilizados com sucesso no projeto de extensão "Reabilitação Protética Buco Maxilo-facial" do Centro Universitário Newton Paiva, tornando-se um aliado pré-clinico fundamental para a preparação dos alunos.
\end{abstract}

Palavras-chave: Reabilitação, Face, Ensino.

\section{ABSTRACT}

The maxillofacial prosthesis is the specialty responsible for feeding intra and extra oral imperfections, congenital or acquired. Facial imperfections compromise some biological and aesthetic functions and can also cause psychological damage. It is extremely important for a multidisciplinary team to analyze the etiology of the problem to which the patient seeks treatment. There is a significant increase in the quality of life of patients with prostheses, indicating the importance of the specialty. The objective of the study is, through an experience report, to describe the development of a unique and exclusive mannequin to assist the training of impression of patients with intraoral defects and consequently spread the teaching of the specialty among the faculties of dentistry. The mannequins created are successfully used in the Maxillofacial Rehabilitation Extension Project at the Centro Universitário Newton Paiva, becoming a fundamental preclinical ally for the preparation of students.

Keywords: Rehabilitation, Face, Teaching 


\section{INTRODUÇÃO}

A busca pela reparação de imperfeições faciais não é algo recente. Há séculos o homem já tentava restaurar imperfeições da face, como se pode observar nas antigas múmias egipcias que possuiam estruturas faciais artificiais (Araújo, Meyer \& Souza, 2009). Diversos tipos de materiais eram empregados na reconstrução facial, como jade, porcelana, madeiras e várias resinas. Nas comunidades gregas e romanas também foram encontrados vestígios protéticos. Reconstruir a face passou a significar o resgate da identidade do individuo. Foi no decorrer da Primeira Guerra Mundial que surgiram especialistas na área de Prótese Bucomaxilofacial (PBMF) (Rosa, 2007; Simões, Reis \& Dias, 2009).

A PBMF é uma especialidade odontológica responsável pela reabilitação facial intra ou extraoral de pacientes com más-formações congênitas, distúrbios de desenvolvimento e deformidades adquiridas em casos de trauma ou patologias (CRO-SP; Araújo, Meyer \& Souza, 2009). Os objetivos da especialidade são devolver a anatomia, a função, a fala, a deglutição, a estética, a recomposição, e a proteção dos tecidos adjacentes por meio de substitutos artificiais. O contorno facial e a cavidade presente devem ser preenchidos ainda que de forma parcial. O fator crucial para reabilitação é a estética da prótese, uma vez que essa aumenta a autoestima e a autoconfiança do paciente, devolvendo, de maneira menos traumática, a reintegração ao convivio social, possibilitando maior qualidade de vida (Rizzati-Barbosa et al., 2003; Araújo, Meyer \& Souza, 2009; Alfenas et al., 2011; Mantri \& Khan, 2012; Aguiar et al., 2013; Batistella, 2015; Prasad, Swaminathan \& Prasad, 2016).

A face é a parte mais visivel do corpo e fornece a identidade a cada um. Funcionalmente, a face promove a expressão, a comunicação, bem como fornece vias de acesso essenciais para o sistema respiratório e gastrointestinal. Cognitivamente, essa região é a única fonte de visão, audição, olfato e paladar, trazendo assim inúmeros problemas quando ocorre a sua desconfiguração congênita ou adquirida, sendo um desses problemas a relação do sujeito acometido com o meio social (Wallace \& Wei, 2008). A cada ano é considerável o número de novos afetados por deformidades faciais (Araújo, Meyer \& Souza, 2009). Os tratamentos de câncer de cabeça e pescoço, por exemplo, têm várias modalidades, incluindo a radioterapia e a quimioterapia para o controle da doença, redução das metástases e a preservação de estruturas anatômicas (Mantri \& Khan, 2012). No Brasil, a ocorrência do câncer bucal é cerca de 15 mil casos por ano (INCA, 2019). A cirurgia é a primeira escolha quando o câncer é detectado prematuramente e é indicada também nos casos em que os tumores não respondem à radiação e à quimioterapia. Caso não tratados, os tumores podem resultar em comprometimentos estéticos, funcionais e psicológicos, afetando muito a qualidade de vida do paciente (Mantri \& Khan, 2012). Sendo assim, as próteses são a primeira alternativa para a correção das imperfeições bucomaxilofaciais, podendo ser complementares às cirurgias plásticas quando estas não obtêm sucesso ou mesmo quando não podem ser realizadas devido 
à idade avançada, à saúde desfavorável, a deformações extensas ou ao inadequado suprimento sanguíneo (Chalian, Drane \& Standis, 1972; Rizzati-Barbosa et al., 2003; Araújo, Meyer \& Souza, 2009; Mantri \& Khan, 2012; Prasad et al., 2016).

Dentre os diversos desafios enfrentados pelo cirurgião dentista que trabalha na área da PBMF, as comunicações bucosinusais são aquelas menos toleradas pelos pacientes, pois podem envolver pequenas porções do palato duro e/ou mole, soalho nasal ou, ainda, comprometer boa parte dessas estruturas bucomaxilofaciais, levando à desnutrição, à perda de peso e causando impacto psicológico negativo nos individuos envolvidos (Goiato et al., 2004). Já os defeitos mandibulares incluem ressecção cirúrgica de mandibula, língua, assoalho da boca e estruturas associadas. Os defeitos também podem prejudicar a fala, dificultar a deglutição, causar desvio de mandibula, trismo, deficiência nas secreções salivares e desconfiguração da face (Mantri \& Khan, 2012). É possivel ainda que haja perda de estruturas extraorais, como olhos, orelhas e nariz (Graziani, 1982).

As imperfeições bucomaxilofaciais podem comprometer não só a estética e a expressão facial, mas também prejudicar algumas funções biológicas do organismo humano, como a fala e a mastigação, provocando consequências negativas para o estado nutricional do indivíduo, além de causarem danos psicológicos que poderão levar à condição de isolamento, em se tratando do convívio em sociedade. Em alguns casos, os pacientes desenvolvem depressão, ansiedade, pânico, isolamento social e crises de autoidentidade (Mantri \& Khan, 2012).

Os recursos e opções de tratamento para esses casos implicaram em progresso para a sobrevida dos pacientes, fazendo com que a reabilitação se tornasse mais urgente e necessária. São procedimentos únicos, uma vez que dependem da demanda de cada paciente (Rizzati-Barbosa et al., 2003; Mantri \& Khan, 2012). É importante conhecer a etiologia do problema para proceder com a terapêutica mais adequada e obter o sucesso na reabilitação. Saber as reais necessidades e reconhecer as expectativas de cada paciente pode ajudar na execução de um bom planejamento protético. Ademais, é imprescindivel que o paciente que irá se submeter à prótese coopere, compreenda e aceite as limitações da reabilitação (Barros, 2017). Uma vez que a prótese possua boa manutenção, pode melhorar a qualidade de vida e fazer com que ocorra a superação do trauma vivido pelo paciente, diminuindo as sequelas que foram deixadas pela deformidade facial (Araújo, Meyer \& Souza, 2009; Aguiar et al., 2013). Avaliar o estado mental e a coordenação motora do paciente para que este mantenha a prótese também é de suma importância para o tratamento (Batistella, 2015).

Na técnica convencional para desenvolvimento da PBMF, a prótese pode ser esculpida levando-se em consideração a anatomia do elemento perdido ou deformado. Esse procedimento é trabalhoso, demorado e depende exclusivamente da habilidade artística do operador. Atualmente, é possivel obter modelos através de escâneres 3D, tomografia computadorizada e ressonância 
magnética. Porém, essas técnicas infelizmente ainda são de alto custo (Aquino et al., 2012).

Em se tratando de materiais protéticos, as propriedades desejáveis desses incluem durabilidade, flexibilidade, biocompatibilidade, baixo peso, estabilidade de cores, higiene, condutividade térmica, facilidade de fabricação e de uso, textura, disponibilidade e baixo custo (Mantri \& Khan, 2012). Vários materiais vêm sendo testados. Os que mais se destacam são o metilmetacrilato, por ser mais resistente, e o silicone, por ser macio e flexível. Porém, nenhum deles é ideal em todos os aspectos (Khan, Gettleman \& Jacobson, 1992).

Os resultados do desenvolvimento das próteses que reabilitam pacientes com perdas de estruturas da região de cabeça e pescoço são extremamente eficazes para a melhora das condições básicas de vida desses pacientes. Esse fato nos mostra a importância de tal especialidade, e a necessidade do seu ensino para os alunos em formação (Alfenas et al., 2011).

As PBMF talvez sejam o maior desafio do profissional dentista, por precisarem não só devolver a anatomia, como reabilitá-la com naturalidade. Bons resultados estéticos dependem desde a escolha dos materiais até dos adequados procedimentos de moldagem e processos laboratoriais para fornecerem resultados satisfatórios às próteses. Esta área exige que os profissionais tenham ampla visão clínica para atuarem com equipes multidisciplinares (Simões et al., 2009). A suposta dificuldade em ser um protesista bucomaxilofacial está bem clara quando se analisa a quantidade de profissionais especialistas registrados, que no caso do Brasil são apenas 62 (CFO). No Brasil, o título de especialista em PBMF é aplicado ao cirurgião dentista. Entretanto, boa parte dos estudantes e dos profissionais de Odontologia desconhecem a área ou apresentam um vago conhecimento sobre ela.

Considerando que os casos de imperfeições faciais estão cada vez mais recorrentes, o desenvolvimento do manequim foi planejado levando-se em consideração o desconhecimento do processo e da dificuldade de moldagem das deficiências intraorais. A moldagem é uma etapa indispensável. Ela não pode ser mal executada, pois é através dela que será obtido o modelo de trabalho para confecção da prótese. Essa fase exige não só conhecimento teórico, mas também habilidades práticas para uma boa execução. Sendo assim, o treinamento constante do procedimento é parte fundamental para o seu sucesso.

Isso posto, o objetivo deste trabalho consiste em, através de um relato de experiência, descrever o desenvolvimento de um manequim único e exclusivo para auxiliar no treinamento de moldagens em pacientes com deficiências intraorais. Esse é o primeiro passo para o desenvolvimento das PBMF. Além disso, busca-se, com este trabalho, tornar a PBMF mais acessivel entre os cirurgiões dentistas e também difundir o ensino da especialidade entre as faculdades de odontologia de maneira mais didática, segura e interativa para os discentes.

Com os manequins que simulam os defeitos intraorais, os alunos podem treinar diversas vezes antes de serem submetidos ao atendimento direto aos pacientes, desenvolvendo, assim, melhor habilidade e confiança no momento 
de realizar as moldagens para confecção das próteses. Com prévias práticas de moldagem das estruturas, o aluno poderá se sentir mais seguro na prática clínica, já que vivenciou um preparatório de métodos de manuseio das cavidades, tomando conhecimento de algumas das possibilidades de deformidades que podem ser encontradas e os diferentes tipos de materiais para serem utilizados em cada caso.

\section{MATERIAIS E MÉTODOS}

Os manequins foram desenvolvidos para serem utilizados no projeto de extensão "Reabilitação Protética Buco Maxilo-Facial" do Centro Universitário Newton Paiva, em Belo Horizonte - Minas Gerais. Eles foram personalizados a partir de um manequim padrão de maxila totalmente desdentada e mandíbula classe II, modificação 3 de Kennedy. Foram criadas as deformidades de acordo com as regiões mais acometidas por tumores orais e que são mais frequentes de serem encontradas clinicamente. Os manequins vêm então sendo utilizados há três anos no projeto de extensão citado, com excelente aplicabilidade no treinamento pré-clínico.

\section{RELATO DE EXPERIÊNCIA}

Uma época em que os defeitos maxilares são cada vez mais comuns é primordial ter conhecimento dos procedimentos existentes, desde a cirurgia até a reabilitação protética (Pereira, 2014).

Não existem, tanto na literatura quanto no mercado, manequins com imperfeições orais e faciais para o treinamento de moldagem em PBMF, o que ressalta o desconhecimento do tema por parte dos profissionais e a falta de estrutura para o desenvolvimento da disciplina nas universidades, pois grande parte das disciplinas práticas, em Odontologia, apresentam um treinamento pré-clínico para preparar o aluno e dar a ele treinamento e confiança adequada antes de realizar o procedimento em pacientes.

Embora existam controvérsias quanto ao momento ideal para a confecção da prótese obturadora definitiva, esta deve ser realizada quando o paciente apresentar condições de aceitação da nova fase de reabilitação que se inicia, além de apresentar a cicatrização completa da área operada, pois, caso contrário, a cavidade poderá sofrer pequenas alterações (Fonseca \& Silva, 2020; Singh et al., 2013). Essas próteses são constituídas por palato, rebordo, dentes artificiais e balão obturador, este dependendo do tamanho do defeito maxilar (Meenakshi \& Shah, 2012).

O manequim foi adquirido através da empresa Nacional Ossos ${ }^{\circledR}$. com maxila totalmente desdentada e mandibula classe II, modificação 3 de Kennedy, ausência unilateral de dentes posteriores, além de dois novos espaços edên- 
tulos (Curtis et al., 1992). Todas as fotos presentes neste relato de experiência possuem como fonte acervo pessoal.

\section{Figura 1}

Manequim sem alterações

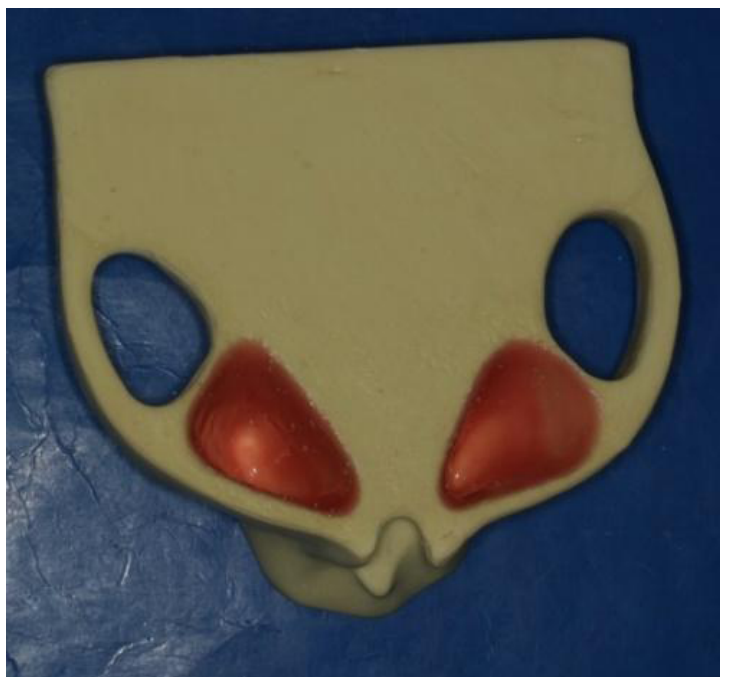

Figura 2

Manequim sem alterações

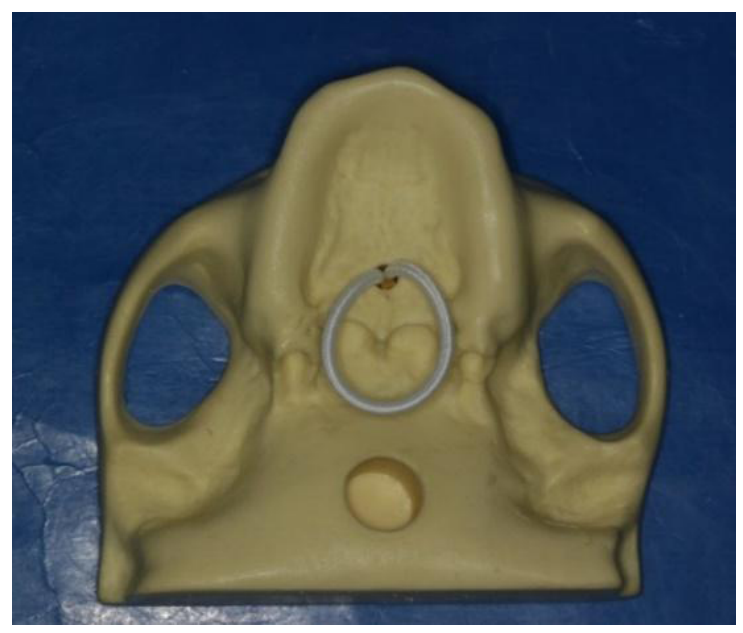


Figura 3

Manequim sem alterações

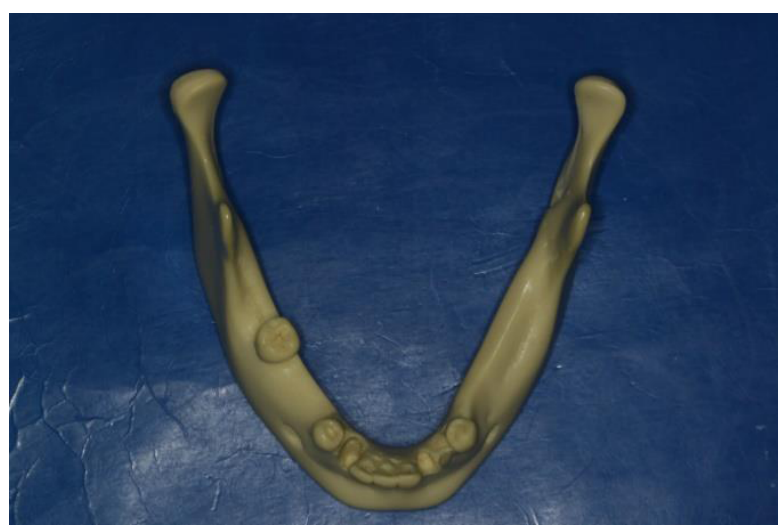

Após a primeira etapa de aquisição dos manequins concluida, o segundo passo foi a reprodução das deficiências intraorais. Uma vez que o manequim se desarticula, a mandibula foi separada do restante da maxila para facilitar a confecção das imperfeições. Para isto, foi marcado no manequim, com uma broca minicut (AMERICAN BURRS ${ }^{\circledR}$ ), até o local em que as irregularidades abrangeriam. Feitas as marcações, o próximo passo foi a utilização da broca maxicut (AMERICAN BURRS ${ }^{\circledR}$ ), tornando possivel que o material do manequim fosse desgastado mais facilmente. Após a remoção completa da área demarcada, as deficiências da maxila e mandibula estavam concluídas e os modelos prontos para a prática em laboratório.

\section{Figura 4}

Processo de confecção dos defeitos com broca minicut

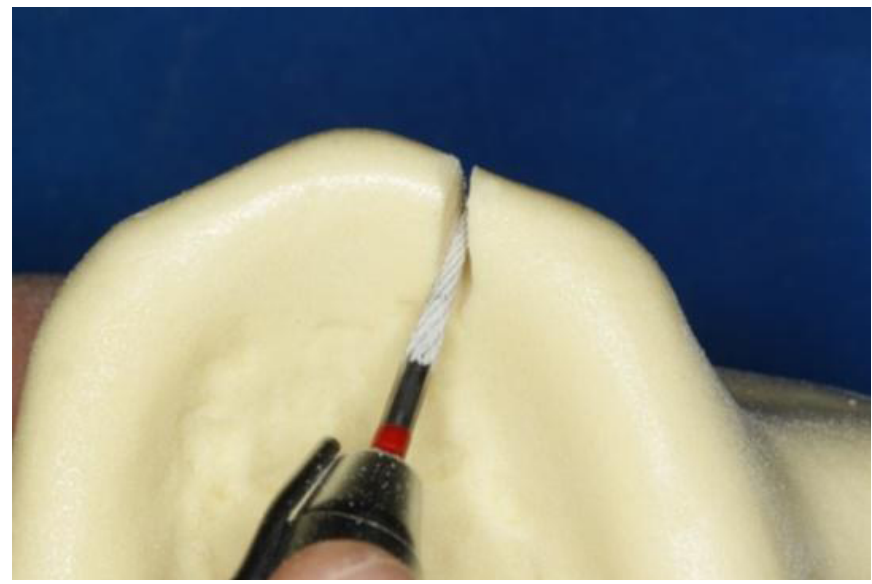


Figura 5

Processo de confecção dos defeitos com broca maxicut

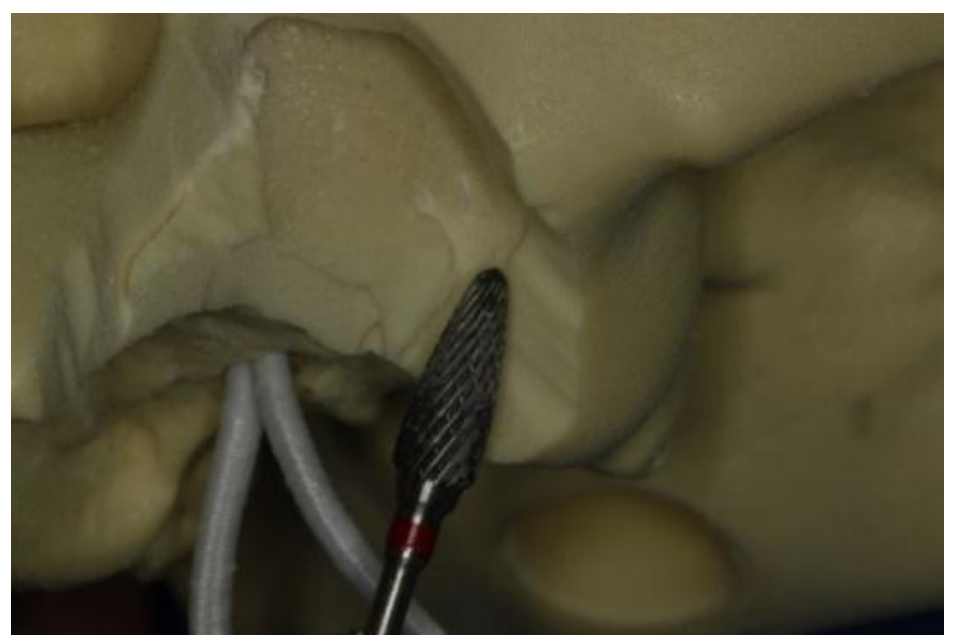

Figura 6

Processo de confecção dos defeitos com broca minicut

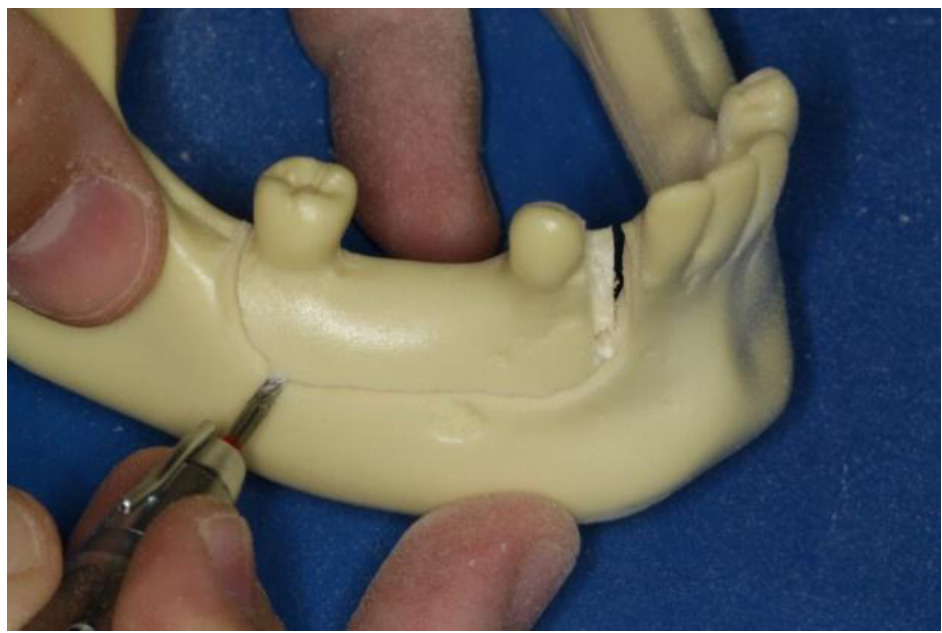

Uma das alterações feitas nos manequins transformou a maxila do lado esquerdo em um defeito classe I de Aramany, pois este é um defeito muito comum em pacientes que apresentam carcinomas intraorais. O defeito classe I de Aramany é aquele que não atravessa a linha média e as estruturas dentárias do lado contralateral. Quando estas estão presentes, são preservadas (Aramany, 2011). O carcinoma adenoide cístico ocorre com uma incidência de $40 \%$ a $45 \%$ na glândula salivar menor, sendo a localização mais comum no palato. Trata- 
se de um tumor maligno que representa de $8 \%$ a 15\% de todas as neoplasias que acometem essa região. As metástases ocorrem em aproximadamente 35\% dos pacientes. Os carcinomas originários no palato ou seio maxilar, geralmente, mostram características radiográficas com evidente destruição óssea, podendo invadir a base do crânio e se disseminar. Isso ocorrendo, são necessárias cirurgias complexas para remoção do tumor. A excisão cirúrgica é normalmente o tratamento escolhido e, muitas vezes, o paciente apresentará uma grande discrepância na área atingida (Neville et al., 2016; Rabelo et al., 2018).

Figura 7

Imperfeições produzidas na maxila

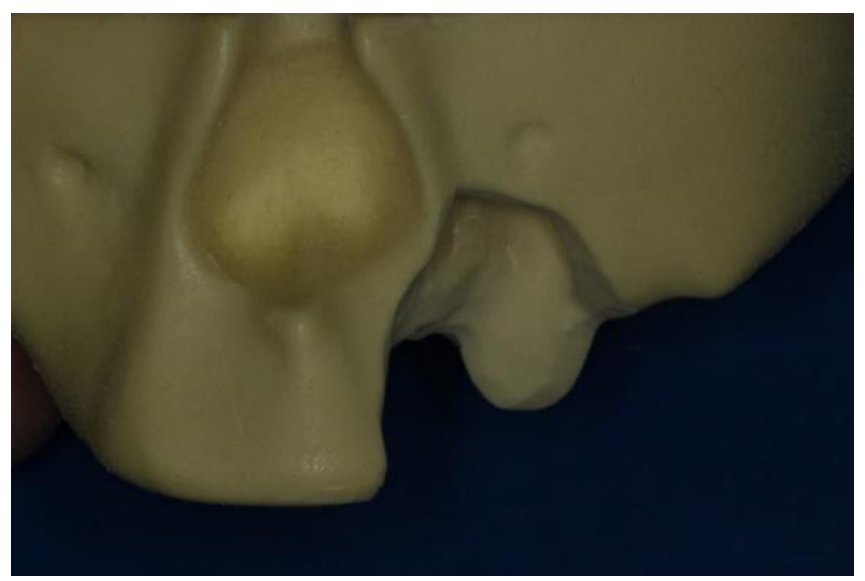

Figura 8

Imperfeições produzidas na maxila

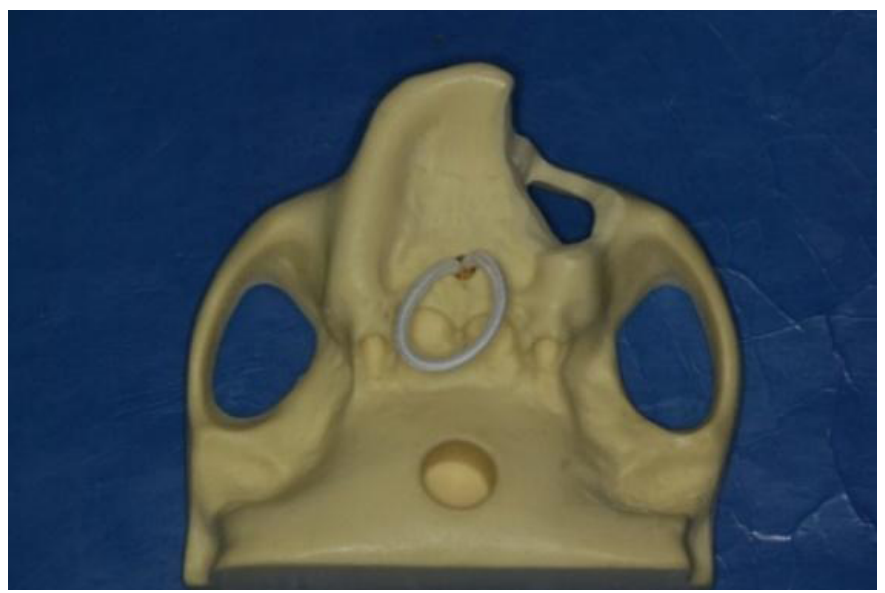


Define-se como maxilectomia, ou ressecção maxilar, a remoção cirúrgica de uma parte ou de toda a maxila, resultando em defeitos denominados maxilares (Brida et al., 2012). No caso, a imperfeição criada no manequim equivale à classificação 3, ou parcial, em que menos de 50\% da maxila foi removida (Sá, 2010). Ainda que, com essa imperfeição, a cavidade oral permaneça funcional e exista a possibilidade de ocorrência de comunicação buco-sinusal. Essas deficiências devem ser corrigidas para evitar o refluxo nasal e outras influências, como, por exemplo, na voz do paciente (Rabelo et al., 2018).

Outra alteração construída no manequim fez com que a arcada inferior se tornasse classe I, modificação 1 de Kennedy, ausência bilateral de dentes posteriores, além de mais um espaço edêntulo, e uma simulação de ressecção segmentar ou marginal do lado direito posterior da mandibula (Curtis et al., 1992). Foi escolhida essa região para confecção do defeito no manequim devido à frequência de acometimento por ameloblastoma, que é o tumor mais comum entre os outros odontogênicos. Essa patologia é uma neoplasia benigna localmente invasiva, normalmente assintomática. Lesões pequenas são detectadas somente através de exames radiográficos. Quando o ameloblastoma é diagnosticado, o tratamento mais aconselhado é a ressecção marginal de mandibula. Entretanto, tem sido observado que a taxa de recidiva é alta. Por isso, alguns cirurgiões dentistas optam por ter uma margem de segurança de um a 1,5cm da margem do tumor (Neville et al., 2016). Em casos em que a lesão já se apresenta extensa, somada à margem de segurança, o paciente pode ter a basilar da mandibula comprometida ou até mesmo totalmente retirada. Nessas situações, o paciente pode desenvolver disfunções na mastigação, assimetria facial e anormalidade dos movimentos mandibulares (Melo, 2016).

Figura 9

Imperfeições mandibulares

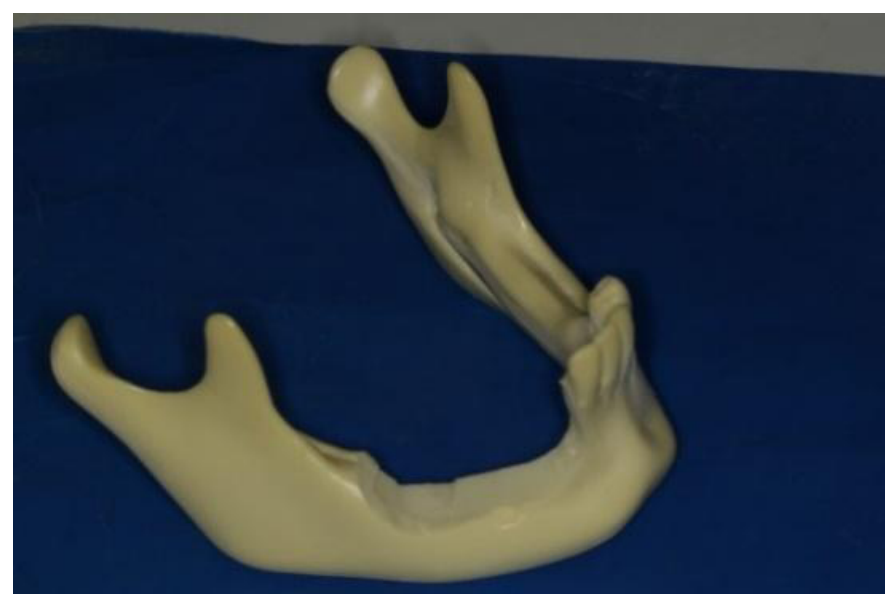


Figura 10

Imperfeições mandibulares

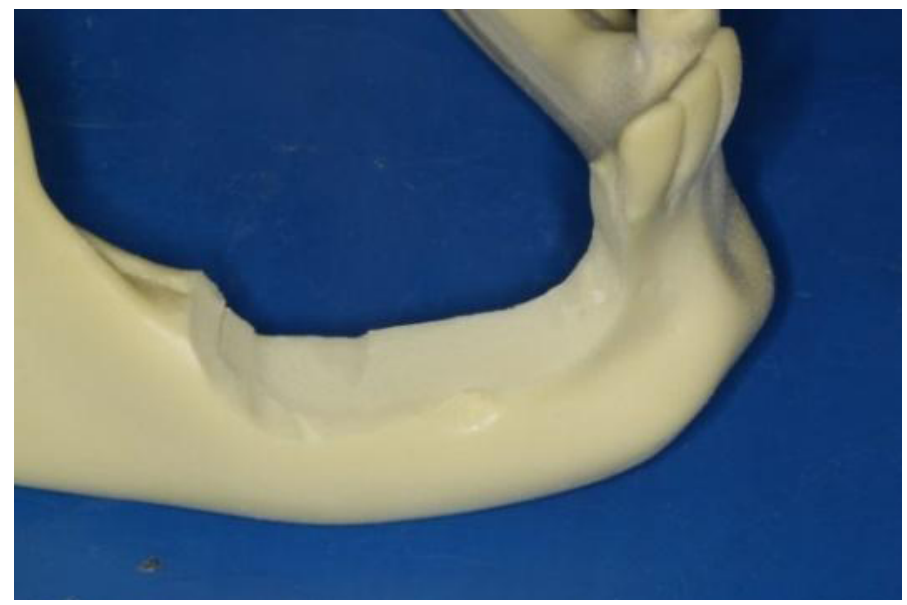

Os manequins vêm sendo utilizados com sucesso há três anos no Projeto de Extensão "Reabilitação Protética Buco Maxilo-facial" do Centro Universitário Newton Paiva em Belo Horizonte, Minas Gerais. O referido projeto de extensão é realizado nesse Centro Universitário desde 2017, tendo a participação de 12 alunos (selecionados anualmente) e 2 professores. O projeto tem como objetivo capacitar o estudante de odontologia para o atendimento do paciente com necessidades de grandes reabilitações maxilo-faciais e, assim, fazer com que esse profissional seja capaz de reinserir socialmente os individuos que apresentam grandes mutilações faciais, intra e extra orais, devolvendo a eles a estética, função e fonação. O público-alvo são pacientes mutilados, após a remoção de tumores na face e aqueles que necessitam de atendimento odontológico, bem como qualquer paciente que apresente alguma deformidade maxilofacial decorrente de trauma, acidente ou patologias, de todas as faixas etárias, gêneros e renda. O projeto apresenta relevante significado para o processo de ensino-aprendizagem dos discentes do Centro Universitário Newton Paiva, pois possibilita o aprendizado de novas técnicas, a revisão de conteúdo de disciplinas pregressas, além de apresentar alto impacto social.

O fato de manequins com imperfeições intraorais não serem encontrados no mercado, ou na literatura científica, exigiu extensa pesquisa sobre como deveriam ser feitos, sobre quais seriam as regiões mais acometidas e sobre quais os tipos de defeitos mais comuns a serem reproduzidos. O processo de confecção gerou altas expectativas quanto a sua capacidade de auxiliar os alunos participantes do projeto nas moldagens.

O primeiro contato dos alunos extensionistas, ainda graduandos, com os defeitos maxilofaciais, acontece por meio desses manequins com o objetivo principal de treinamento de moldagens, apontado como uma das maiores dificuldades na confecção das PBMF. Os alunos têm aulas teóricas sobre os 
temas, treinamentos laboratoriais pré-clínicos, bem como atendimento clínico em pacientes com as necessidades supracitadas. Atender o primeiro paciente portador de um defeito intraoral requer um breve treinamento não só da técnica de moldagem e passo a passo, mas também do material mais indicado para cada caso, o que os manequins proporcionam. Além de permitirem erros, o que no paciente poderia não ser tolerado devido às suas limitações. Os alunos do projeto de extensão julgam de suma importância o treinamento em manequins, visto que os casos possuem características incomuns, o que causa certo receio no atendimento clínico.

Figura 11

Manequim articulado

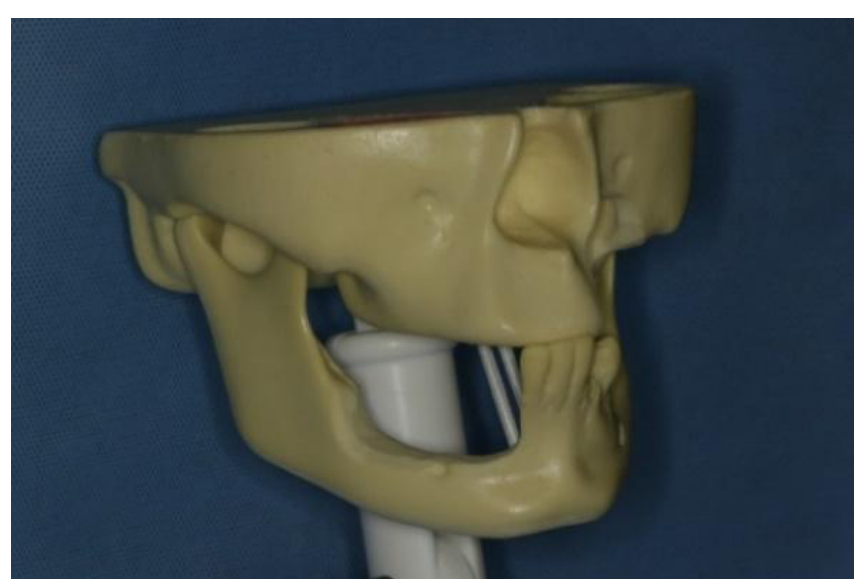

Figura 12

Imperfeições mandibulares

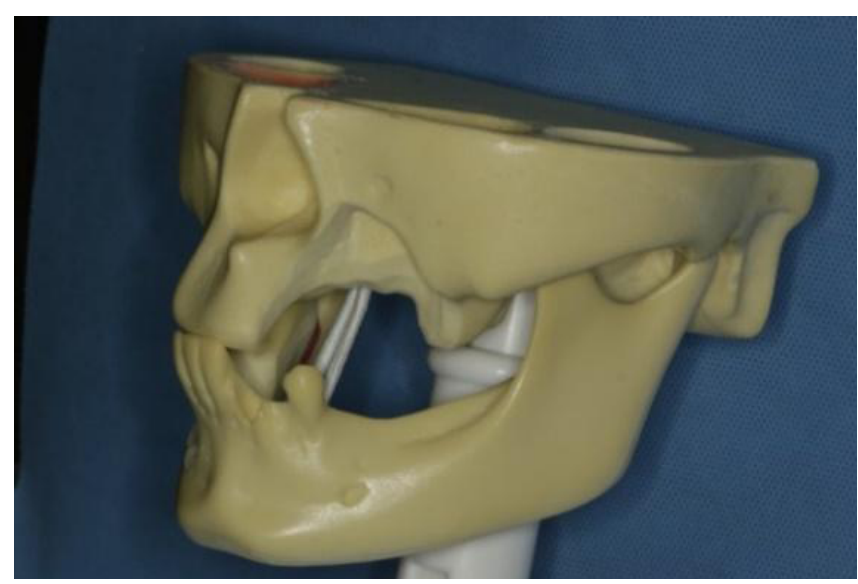


Em geral, as disciplinas do curso de odontologia - como dentística, periodontia, cirurgia e prótese - são introduzidas por um processo pré-clínico. As atividades práticas são um grande destaque na metodologia de ensino. Essas são realizadas através da simulação dos procedimentos odontológicos, fazendo com que os alunos sintam-se mais confiantes e preparados para realizar os procedimentos em pacientes. Na utilização de manequins para treinamento das técnicas, o aluno é estimulado a desempenhar as atividades para que chegue à clínica com um bom desempenho preparatório.

Há uma estimativa de 14.700 novos casos de câncer oral no Brasil, sendo 11.200 em homens e 3.500 em mulheres (INCA) mostrando cada vez mais a necessidade das PBMF. Muitas vezes essas são as únicas alternativas para a resolução dos problemas de imperfeições faciais ou intraorais.

A partir dessa experiência, observamos que os manequins são ferramentas didáticas fundamentais para o ensino e treinamento da especialidade de prótese bucomaxilofacial. Através deles os alunos se mostraram mais estimulados e envolvidos com o procedimento a ser realizado, além de ser uma forma mais segura e aplicável de preparação do aluno para um procedimento pouco realizado e conhecido, que é a moldagem de imperfeições intraorais. Futuramente, novos manequins, com diferentes imperfeições, também podem ser desenvolvidos com o mesmo objetivo e benefício às PBMF.

\section{CONSIDERAÇÕES FINAIS}

A criação dos manequins com imperfeições intraorais para treinamento de moldagens torna-se um grande aliado pré-clínico para a disciplina de PBMF, uma vez observado que até então não havia modelos de aprendizado prático que antecedessem o atendimento a esses pacientes. Aprender a reabilitar alterações anatômicas que uma cavidade oral pode conter é algo desafiador, já que a grande maioria dos pacientes dessa especialidade não apresentam estruturas bucais favoráveis comuns às próteses dentárias convencionais.

A confecção e utilização dos manequins foi uma experiência única no ensino da PBMF, pois através deles os alunos passaram a entender mais sobre as deformidades intraorais, por que elas acontecem, quais são as mais prevalentes, como refletem na vida do paciente e, especialmente, como os cirurgiões-dentistas podem auxiliar na melhora da qualidade de vida de quem é portador de uma imperfeição intraoral. O instrumento criado para auxiliar dentistas e alunos no planejamento e execução de uma reabilitação protética de elevado nível de dificuldade mostrou-se eficaz, tanto do ponto de vista técnico quanto do ponto de vista psicológico, pois através dos treinamentos realizados os discentes se mostraram mais confiantes e preparados para a execução dos procedimentos clínicos. Os resultados obtidos nessa experiência foram extremamente positivos e impulsionam os pesquisadores a buscarem novos métodos e materiais para auxiliarem na evolução dos profissionais. 


\section{REFERÊNCIAS}

Aguiar, L. (2013). Obturador palatino: confecção de uma prótese não convencional - relato de caso. Revista da Faculdade de Odontologia da UPF, 18(1), 125-129.

Alfenas, E. R., Lanza, C. R. M., Barreiros, I. D., Novaes Junior, J. B., \& Mendonça, L. L. (2011). Reabilitação protética do paciente com perda de substância na região de cabeça e pescoço. Arquivos em Odontologia, 47(2), 28-31.

Aquino, L. M. M., Oliveira M., Martins A. P. V. B., \& Barbosa C. M. R. (2012). Técnicas de moldagem da máscara facial. Revista de Odontologia da UNESP, 41(6), $438-441$

Aramany, M. (2001). Basic principles of obturator design for partially edentulous patients. Part I: Classification. The Journal of Prosthetic Dentistry, 86(6), 559-561.

Araujo, C. R., Meyer, G. A., \& Souza, I. A. (2009). Prevalência de Próteses Buco Maxilo Faciais na Faculdade de Odontologia da Escola Bahiana de Medicina e Saúde Pública em Salvador, Bahia. Revista Portuguesa de Estomatologia, Medicina Dentária e Cirurgia Maxilofacial, 50(3), 133-139.

Barros, H. R. (2017). Reabilitação por prótese bucomaxilofacial em palato e região óculo-palpebral após cirurgia oncológica - relato de caso [Trabalho de Conclusão de Curso em Odontologia - Universidade do Estado do Amazonas, Manaus].

Batistella, E. A. (2015). Silicones utilizados em próteses bucomaxilofaciais extraorais: uma revisão de literatura, 2015 [Trabalho de Conclusão de Curso em Odontologia - Universidade Federal de Santa Catarina, Santa Catarina].

Brida, A. S., Jacob, R. F., \& Taylor, T. D. (2012). Classification of maxillectomy defects: a systematic review and criteria necessary for a universal description. The Journal of Prosthetic Dentistry, 107(4), 261-270.

CFO-Conselho FederaldeOdontologia. Quantidade Geralde Cirurgiões-Dentistas Especialistas. https://website.cfo.org.br/estatisticas/quantidade-geral-de-cirurgioes-dentistas-especialistas/.

CRO - Conselho Regional de Odontologia de São Paulo. Prótese Bucomaxilofacial. http://www.crosp.org.br/camara_tecnica/apresentacao/20.html.

Curtis, D. A., Curtis, T. A., Wagnild, G. W., Finzen, F. C. (1992). Incidence of various classes of removable partial dentures. Journal of Prosthetic Dentistry, 67(5), 6647. doi: 10.1016/0022-3913(92)90167-9.

Demir, N., Cevik, P., Okutan, Y., Ozturk, A. N., \& Colpan, B. A. (2015). Different wax sculpture technique for implant-retained auricular prosthesis. European Journal of Dentistry, 9(3), 433-437. 
Fonseca, R. C. A., \& Silva, F. B. (2020). Vedamento de comunicação buco-sinusal com prótese obturadora palatina: relato de caso. Revista Eletrônica Acervo Odontológico, 1, e 3117. https://doi.org/10.25248/reaodonto.e3117.2020

Goiato, M. C., Mancuso, D. N., Fernandes, A. U. R., \& Dekon, S. F. C. (2004). Estudo sobre as causas mais frequentes de perdas oculares. Arquivos em Odontologia, 40(3), 207-286.

Graziane, M. (1982). Prótese Maxilo-Facial. In Graziane, M. Generalidades. Rio de Janeiro: Guanabara Koogan. Cap. 1, 1-4.

INCA - Instituto Nacional de Câncer. Tipos de Câncer: Câncer de Boca. https:// www.inca.gov.br/tipos-de-cancer/cancer-de-boca.

Khan, Z., Gettleman, L., Jacobson, C. (1992). Conference report: Material research in maxillofacial prosthetics. Journal of Dental Research, 71(8), 1541-1542.

Kusterer, L. E. F. L., Paraguassu, G. M., Silva, V. S. M., \& Sarmento, V. A. (2012). Reabilitação com obturador maxilar após cirurgia oncológica: relato de casos. Revista de Cirurgia e Traumatologia Buco Maxilo Facial, 12(4), 9-16. Disponivel em: http:// revodonto.bvsalud.org/scielo.php?pid=S1808-52102012000400002\&script=sci_arttext

Lima, J. G. S. et al. (2012). Reabilitação protética de pacientes com defeitos maxilofacais. In V SEREX - Seminário de Extensão Universitária do Centro-Oeste, 5, 186.

Mantri, S., \& Khan, Z. (2012). Prosthodontic Rehabilitation of Acquired Maxillofacial Defects. Head and Neck Cancer.

Meenakshi, A, Shah, D. (2012). The obturator prostheses for maxillectomy. SRM Journal of Research in Dental Sciences, 3(3), 193-197.

Melo, R. M., Carneiro, N. C. M., Fonseca, W. L. M., Lima, J. F., Araujo, H. P. S., \& Pontes, H. A. R. (2016). Tratamento cirúrgico de ameloblastoma sólido convencional: relato de caso clínico. Revista da Faculdade de Odontologia da UPF, 21(2), 246-250.

Neville, Brad W. et al. (2016). Patologia oral e maxilofacial. 4 ed. Rio de Janeiro: Elsevier, 912.

Pereira, A. M. L. (2014). Reabilitação Oral em Pacientes Maxilectomizados [Dissertação de mestrado em Medicina Dentária - Faculdade de Ciências da Saúde, Universidade Fernando Pessoa Porto].

Prasad, K, Swaminathan, A. A., \& Prasad, A. (2016). Fabrication of a Silicone Auricular Prosthesis: A Case Report. Nitte University Journal of Health Science, 6(1), 91-97. 
Rabelo, D. P., Tanure, R. P., Grajeda, F. M. C., \& Grossmann, S. M. C. (2018). Reabilitação protética oral em paciente oncológico. Revista da Universidade Vale do Rio Verde, 16(1), 1-8.

Ravuri, R., Bheemaligeshwarrao, Tella, S., \& Thota, K. Auricular prosthesis-a case report. Journal of clinical and diagnostic research, 8(1), 294-296. https://doi. org/10.7860/JCDR/2014/7943.3986.

Rizzatti-Barbosa, C. M., Souza F. R. A., Barbosa, J. R. A., \& Souza, E. V. (2003). Prótese Auricular em Resina Acrilica Polimerizada com Energia de Microondas. Revista Brasileira de Prótese Clínica \& Laboratorial, 5(27), 389-393.

Rosa, F. G. (2007). Fixação de prótese facial em pacientes oncológicos: Revisão sistemática [Dissertação de mestrado em Odontologia - Faculdade de Odontologia, Universidade Federal de Urbelândia, Uberlândia].

Sá, S. P. (2010). Próteses obturadoras para pacientes maxilarectomizados: estado atual da tecnologia e necessidades de aprimoramentos [Dissertação de mestrado em Gestão de Tecnologias em Saúde - Instituto de Medicina Social, Universidade do Estado do Rio de Janeiro, Rio de Janeiro].

Silva, D. P., Vaccarezza, G. F., Cazal, C., Almeida, F. C. S., Brandao, T. B., Durazzo, M. D., Caroli, A., \& Dias, R. B. (2004). Reabilitação Protética de Pacientes Maxilectomizados: uma Contribuição da Odontologia e um Convite à Reflexão. Pesquisa Brasileira em Odontopediatria e Clinica Integrada, 4(2), 125-130.

Simões, F. G., Reis, R. C., \& Dias, R. B. A. (2009). Especialidade de prótese bucomaxilofacial e sua atuação na Odontologia. Revista Sul-Brasileira de Odontologia, 6(3), 327-331.

Singh, M., Bhushan, A., Kumar, N., \& Chand, S. (2013). Obturator prosthesis for hemimaxillectomy patients. National journal of maxillofacial surgery, 4(1), 117120. https://doi.org/10.4103/0975-5950.117814.

Wallace, C. G., \& Wei, F. (2008). The Current Status, Evolution and Future of Facial Reconstruction. Medical Journal, 31(5), 441-449.

Yoshida, H., Furuya, Y., Shimodaira, K., Kanazawa, T., Kataoka, R., \& Takahashi, K. (2000). Spectral characteristics of hypernasality in maxillectomy patients. Journal of Oral Rehabilitation, 27(8), 723-730.

Data de submissão: 13/04/2020

Data de aceite: 18/11/2020 